\title{
Application of Image Recognition Technology in Sports Competition
}

\author{
Fengzhi Zhao \& Feng Bao \\ Applied Technical College, Daqing Petroleum Institute, Qinhuangdao 066004, China \\ The research is supported by Educational Commission of Heilongjiang Province of China (2004.3).
}

\begin{abstract}
This article introduces the design principle and implementation method of the automatic judgment software of terminal in sports competition. Image recognition technology makes the competition management level achieve equality, justice, precision and high efficiency, This actualizing automatic judgment by means of recognition model for sports imaging, which is based on the principle of radio frequency identification (RFID).
\end{abstract}

Keywords: Image Recognition, Judgment management software, Sports imaging, RFID

\section{Introduction}

With the rapid development of computer technology and internet, the electric information technology has applications in every walk of life and particularly the sports domain. Some scholars apply high technology measures taking the computer technology as their basis in physical training. This revolutionary change has affected the whole athletic sports. In traditional sports competition, this information gathering mode relied on the personnel giving out judgments through visual judgement then inputs the date recorded into the computer. This process wastes time and energies and may produce various wrong data because of various artificial mistakes which can influence the reliability of information gathered. However, the automatic identification technology can provide automatic transmission of information and automatic identification through computer, and increase the speed and veracity of judgement, make people cast off multifarious works of statistical identification, this eliminate the interference of human judgement, and highly enhances the efficiency and level of sports judgment, and really achieves equity, justice, and a high efficiency for competition.

Presently, the national and domestic sporting competitions, so with computer and electric equipment becoming the important management measures particularly for the track meets which make competition more justice, high efficiency, exact and without errors, however these systems cost very expensively (usually about millions of yuan RMB). General universities, middle schools, elementary schools and other grass roots units can not support these large investments at all, and their sports technology is still low, and most of them have no electronic equipment for training and competition. To fulfill the needs of training and competition of grass roots athletic meeting, in this article we adopt video frequency and radio frequency identification technology to realize automatization of operation which needs personnel in the past, and this system we designed has many characters such as high precision, low costs, convenient use and will be more accurate for providing good judgements in sporting events. - whether a large meet or a grass-roots meet. All will be fair in the world of sports.

\section{Design principle of system}

\subsection{Main operation principle}

When the competition begins, the starting camera gathers the light and smog produced by the starting gun and inputs them into the computer to be the sign point of timing start. In the competition, the tracking camera is fixed beside the finish line, which follows and gathers the images of every competition, and watches problems occurring in the process of competition. The terminal camera is fixed beside the finish line, which shoots the images of terminal sprint process of every competition following inclined angle of vertical plane. To the competition category by course, firstly, the image gathering system records and stores the video information when every group of athlete passes terminal, then the computer automatically distinguishes the video image that the chest of every athlete achieves the terminal according to the course and compute the competition achievement of every athlete. To the competition category without course, because every athlete takes the sign of RFID, so the RFID reader can transmit the information that every athlete passes the terminal every time to the computer and the computer will record the athletes' identity information and the time that the athletes pass the terminal every time. When the competition ends, the computer will automatically compare with the information gathered by the starting camera, and give out every athlete's grade and place.

The whole system is composed by image gathering system, image identification processing system, athlete data 
management system, RFID radio data processing system and network system. Hereinto, the image gathering system includes camera, video gathering card and video gathering and processing software, which mainly gathers the video information (firing screen) of the starting and the video information of the terminal in every course to the computer and stores them according to their respective course appellation and group. The image identification processing system applies the image processing software to establish image processing platform, and its main function is to identify and time the video images of sprint process gathered by terminal camera. When the competition grades are very close in short distance competition, the grades can be judged through the single frame in a sequence by the video return technology, then the data of place and time affirmed will be transmitted to central management computer for output and storage. The RFID radio data processing system receives the data transmitted by RFID reader and completes lap record and totalizing timing in the middle and long distance competitions. Finally the athlete data management system applies system management software to implement schedule arrangement, grade processing, place ranking, data output and storage for the competition.

\subsection{Design of software module}

The software design of this system includes video image gathering system, video image processing system and radio information processing system.

The video image gathering system has functions including starting shoot, stopping shoot, storing video image and video return.

The video image processing system has functions including establishing athlete information, inputting checking list, video processing and timing, picking up final list, clearing checking list, initializing system and setting up system.

(1) Establishing athlete information. Input athletes' basic information (athlete number, name, sex, group and course) to the database. It also includes some sub-functions such as adding, deleting, inquiring, printing and saving.

(2) Inputting checking list. In the competition, according to starting checking information pick up or add every athlete's information such as number, name, course and group from the database to the checking list, and offer information for the timing processing module.

(3) Video processing and timing. Move the video files saved as "course + group" by the video gathering system to the computer which will deal with these images. First, confirm the starting time through identifying firing screen, then form instantaneous continuous narrow digital images of sprint, move the vertical time axis line to certain part (regulated by the competition rule) of the mobile, push mouse key to confirm, so the computer will read out grades and automatically rank, finally, store videos after processing to the hard disk or mobile disk. This function also includes quick orientation of video, single frame return, course identification, grade computation, results printing and saving data.

(4) Clearing checking list. Completely delete all information in the present checking list and prepare for the next event.

The radio information processing system includes functions of establish initial information of every athlete, starting reader, lap record processing and comprehensive timing processing.

(1) Establishing initial information of every athlete. Establish corresponding relations between digital information on the RFID sign and every athlete's number in every group.

(2) Comprehensive timing processing. Record the totalizing time that athlete passes the terminal every time. When the competition ends, compute every athlete's grade through comparing with the starting time confirmed by the video identification processing.

\section{Key technology and implementation method}

To realize automatic judgment through image identification technology, we need solve following several key problems including (1) how to confirm the starting time, (2) how to identify the sprint video of athlete, (3) how to integrate the videos gathered by the starting camera and the terminal camera, (4) how to compute athletes' grades, (5) how to timing in the middle and long distance events?

Hereinto, the most pivotal technologies are the confirmation of starting time and the identification of sports image which will be discussed in the following text.

\subsection{Confirmation of starting time}

In the initial stage of system design, all methods to confirm the starting time are to add a switch quantity, then transmit the analog signals to the central signal controller through communication cable, and the controller receives and deals with the electric signal of starting point and transform it into digital signal and input it into the computer which will start timer of zero clock, so the starting time is confirmed. This method can timely and exactly obtains the starting signal, but to enhance the reliability of the system, we need buy equipments with high performance and equip a circuit communication cable along the playground, so the costs are very expensive and it is not fit for grass roots games. Through large numbers of experiments, we can find that the video of firing screen also can timely and exactly confirm 
the starting time, and the costs are cheap and the use is convenient (Figure 1 is a group of firing video gathered where the first frame is the image to confirm the starting time).

The implementation method can be described as follows. First, obtain the background image in the initial situation of firing screen, then make subtracting operation between present input image and the saved background image and obtain the difference image (seen in Figure 2). According the number of pixel point that the grey degree is bigger than certain threshold value in the difference image, we can confirm the first frame in the firing screen and take is as the start of timing. The concrete implementation flow is seen in Figure 3.

\subsection{Video identification principle of athletes'sprint}

\subsubsection{Basic principle of video identification}

(1) Adopting the Gaussian filtering method to filter the serial frame difference of image, strengthening the robustness of frame difference to the yawp and automatically separating the sprint area and the background.

(2) According to three frames of image in the image sequence, effectively solving the sheltered problem the former frame and the later frame of sprint through taking the edge superposition of the image grey in the first and last frames difference as the edge of the middle frame.

(3) Adopting joint area operator, taking the joint area which area is smaller than certain threshold value as the background, accordingly eliminating big background yawp and labeling sports multi-area.

(4) To every sports area labeled, adopting outside profile tracking arithmetic to automatically position the initial profile of mobile object, and adopting improved dynamic profile shrinking arithmetic to exactly confirm the outside profile of the object and extract the profile of sports multi-objects.

The checking model of sprint area can be described as follows.

$$
\begin{aligned}
& D f(x, y)_{[t, t+1]}=G(x, y) \times|f(x, y, t+1)-f(x, y, t)| \\
& B D f(x, y)_{[t, t+1]}= \begin{cases}1 & \text { if } \quad D f(x, y)_{[t, t+1]}>\tau \\
0 & \text { otherwise }\end{cases} \\
& \text { Where, } \tau=\operatorname{mid}\left(\tau_{1}, \tau_{2}, \tau_{3}, \tau_{4}\right) \\
& \tau_{i}=\frac{1}{N_{M_{i}}}\left[c \cdot \sum_{\left(u, v \in M_{i}\right)} D f(u, v)_{[t, t+1]}\right] \cdot
\end{aligned}
$$

\subsubsection{Profile identification model of sports multi-objects}

The dynamic profile model can be described as follows.

$v(s)=(x(s), y(s)) \quad[s \in[0,1]]$

And the energy function in the dynamic profile can be described as follows.

$$
\begin{aligned}
& E_{\text {total }}=\int_{0}^{1} E\left((v(s)) d s=\int_{0}^{1}\left[E_{\text {int }}(v(s))+E_{\text {ext }}(v(s))\right] d s\right. \\
& \left.E_{\text {total }}=(v(s))=(\alpha(s))\left|v_{s}(s)\right|^{2}+\beta(s)\left|v_{s s}(s)\right|^{2}\right) \\
& E_{\text {ext }}(v(s))=-\gamma|\forall f(v)|^{2}
\end{aligned}
$$

\subsection{Timing in middle \& long distance competition events by RFID technology}

In the middle and long distance competition events, because of too many athletes, the competition rule without course is adopted. The usage of video identification technology has following problems. The first problem is that the technology only can confirm the grade that the athlete passes terminal, and can not confirm concrete athlete. The second one is that it is difficult to identify retaining lap. To solve above problems, this system adopts the technology of RFID.

The basic composing of RFID includes tag, reader and antenna. The tag is composed by coupling component and chips, and every tag possesses one and only electric coding attached on the object marking object. The reader can read (sometimes read-in) the information of tag, which can be designed as hand type or fixed type. The antenna transmits radio signals between tag and reader.

The key technology is to tie the radio card (tag) on the athlete's shoestring and place little shim block with mini-antenna on the terminal of the event (seen in Figure 4). The reader can read and identify the athletes' data saved in the radio card through an untouched way accordingly the automatic identification of athletes' information can be achieved. Connecting the reader with the computer, the information read is transmitted to the computer for the next processing. 
When athlete passes this shim block every time, the timing system will receive ID number sent by radio card on the athlete and record the present time and passed lap number.

RFID is a sort of automatic untouched identification technology, which automatically identifies the object through radio signal and acquires relative data, and because the identification needs not manual work, so it can work under various environments. The technology of RFID can identify mobile object with high speed and multiple tags at the same time, and the operation is quick and convenient.

\section{Conclusion}

In this article, we discuss the key technology and implementation method that we adopt image identification technology to actualize the automatic judgment in the athletic sports, and the practice indicates that the resolution of computer image position in this system is 25 frames per second and the time resolving is $4 \%$ second which is synchronized with the identification menu. The grade time is very exact according with the standard of International Association of Athletics Federations (IAAF). The image identification timing judgment at terminal in the event can be implemented timely, delayed or repeatedly, which doesn't influence the starting time and can ensure the event is implemented successfully. The competition process is automatically managed by the program of computer and the judgment personnel are very few, so the computer can automatically complete the mission of terminal judgment. The video material of the whole competition can be saved automatically, and the disputed grades or problems can be discussed conveniently, and various man-made mistakes in the competition can be eliminated, so the judgment level of the competition can be enhanced and the justice, speediness, nicety and high efficiency of the competition can be ensured.

\section{References}

Cai, Wandong. (2000). Multimedia Communication Technology. Xian: Publishing House of Xi'an University of Electronic Science and Technology.2000.10. p.50-99.

Cao, Zhijin \& Tang, Huiming. (2004). Identification of Auto Type in Video Image. Computer Engineering Application. No.24. p.226-228.

D.Zhong and S. F. Chang. (2001). Structure Analysis of Sports Video Using Domain Models (C). IEEE Conference on Multimedia and Exhibition. Tokyo, Japan. Aug, 2001. p.22-25.

Gunilla Borgefors. (1986). Distance Transformations in Digital Images. Computer Vision, Graphics, and Image Processing. No.34. p.344-371.

Wangqian. (1999). Judgment Manual of Athletics Competition. Beijing: China People's Publishing House of Sports. p.81-140.

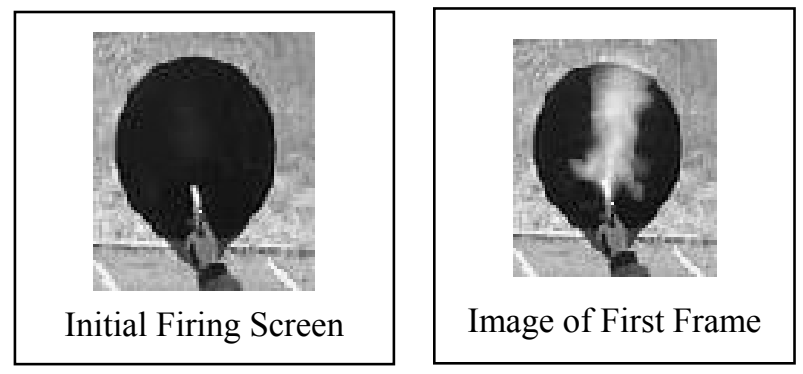

Figure 1. Changes of Firing Screen Video

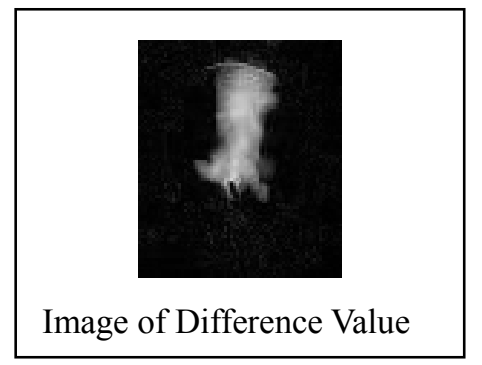

Figure 2. Image of Difference Value 


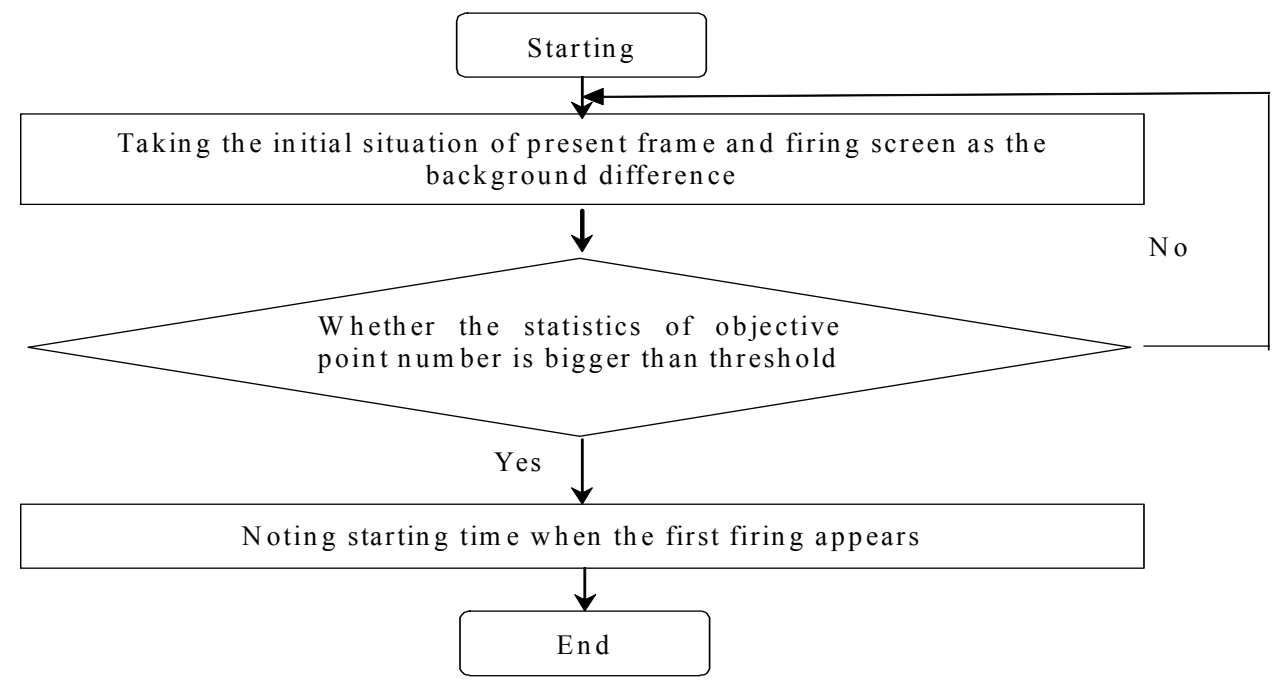

Figure 3. Flow of Starting Time Identification

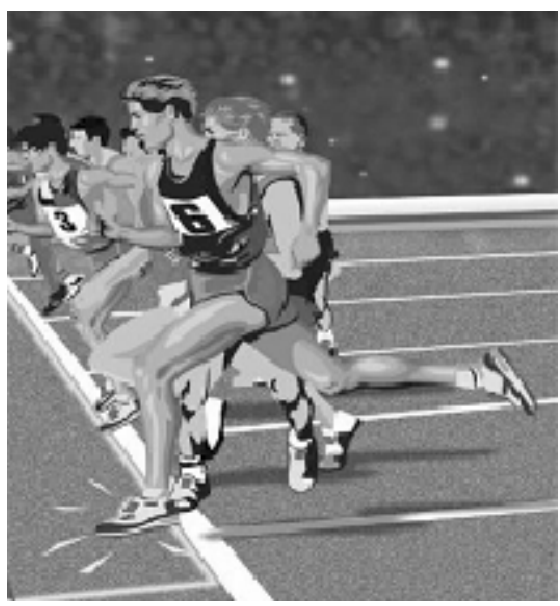

Figure 4. Little Shim Block of REID Mini Antenna 